\title{
Highly Regioselective Synthesis of Novel 1,4'-Bipyrazoles
}

\author{
Marcos A. P. Martins, * Gabriela F. Fiss, Clarissa P. Frizzo, Fernanda A. Rosa, \\ Helio G. Bonacorso and Nilo Zanatta \\ ${ }^{a}$ Núcleo de Química de Heterociclos, Departamento de Química, \\ Universidade Federal de Santa Maria, 97105-900 Santa Maria-RS, Brazil
}

\begin{abstract}
Este trabalho descreve a síntese de 1,4'-bipirazóis inéditos pela da reação de ciclocondensação de novas $\beta$-dimetilaminoenonas com as monoidrato de hidrazina, tert-butil hidrazina, fenil hidrazina, carboximetilhidrazina. Os produtos foram obtidos com alta regiosseletividade em rendimentos de bons a moderados (50-80\%). A técnica de cristalografia de raios-X foi utilizada para a elucidação da regiosseletividade da formação dos produtos. 1,4'-Bipirazolilisoxazóis também foram descritos.

Novel 1,4'-bipyrazoles were synthesized via highly regioselective cyclocondensation reactions of $\beta$-dimethylaminoenones with hydrazine monohydrate, tert-butylhydrazine hydrochloride, phenylhydrazine hydrochloride or carboxymethylhydrazine. X-ray diffraction technique was used in the elucidation of the regiochemistry of the $\beta$-dimethylaminoenaminones and of the 1,4'-bipyrazoles. 1,4'-Bipyrazolylisoxazoles were also reported.
\end{abstract}

Keywords: enaminones, bipyrazoles, heterocycles, cyclocondensation, regioselectivity

\section{Introduction}

Many heterocyclic compounds have interesting biological activities. Pyrazoles and isoxazoles figure prominently in this regard. ${ }^{1}$ The recent success of a pyrazole COX-II inhibitor has further highlighted the importance of these heterocycles in medicinal chemistry. ${ }^{2}$ The past few years have also witnessed explosive developments in combinatorial chemistry and solid-phase synthesis. Currently, there is a particular interest in bipyrazoles substituted in studies on intermolecular interactions with the participation of pyrazoles and their derivatives. ${ }^{3-9}$ The structure of pyrazole molecules, containing the fragment $\mathrm{RN}-\mathrm{N}(\mathrm{H})-$, is favorable in the formation of various hydrogen bonded complexes and associates in solid-cyclic dimers, trimers and tetramers, linear chains and helices, depending on the substituent. ${ }^{9-11} \mathrm{~A}$ literature survey turned up a few reports on 1,4'-bipyrazole and isoxazole synthesis and structure. ${ }^{12}$ The most promising method is a four-steps synthesis via the cyclization of an $\alpha$-oxo- $\alpha$-(3,5-dimethyl$1 H$-pyrazole-1-yl ketene dithioacetal with hydrazine monohydrate or phenylhydrazine in refluxing ethanol. ${ }^{13}$ $1,4^{\prime}$-Bipyrazoles have been also prepared by the reaction

*e-mail: mmartins@ base.ufsm.br of 2-pyrazolyl-3-dimethylamino acrylate and acrylonitrile with hydrazine monohydrate and phenylhydrazine hydrochloride under microwave irradiation conditions. ${ }^{14}$ Our research group has been dedicated to the development of synthetic routes to heterocyclic compounds for around twenty years. ${ }^{15}$ We have extensively explored the potential of enones such as: 4-alkoxy-1,1,1-trihalo-3-alken-2-ones ${ }^{15}$ and enaminones ${ }^{16}$ as building blocks in cyclocondensation reactions. Our results have shown that the obtainment of heterocycles with high regioselectivity is generally dependent on the structure of the building block and sometimes it is dependent on the reaction conditions. Now we are interested in finding an alternative route to 1,4'-bipyrazoles and 1,4'-bipyrazoylisoxazoles. In view of the formation of new bonds $C-C$ or $C-N$ especially in the 4-position of the pyrazolyl ring, herein we reported our investigation in the regioselectivity of the cyclocondensation reactions of $\beta$-dimethylaminoenones with different 1,2-dinucleophiles such as hydrazine monohydrate, tert-butylhydrazine hydrochloride, phenylhydrazine hydrochloride, carboxymethylhydrazine and hydroxylamine hydrochloride by a three-steps procedure (alkylation, condensation and cyclocondensation reactions, respectively) to obtain the respective azoles in good yields. (Scheme 1). 


\section{Results and Discussion}

The first step involves the synthesis of 2-(3,5-dimethyl$1 H$-1-pyrazolyl)acetophenone $\mathbf{2 a}$ in (71\% yield) and 3-(3,5-dimethyl-1H-1-pyrazolyl)acetophenone $\mathbf{2 b}$ (in $75 \%$ yield) through the alkylation reactions of 3,5-dimethyl-1 $H$-pyrazole with 2-bromoacetophenone 1a and 2-bromopropiophenone 1b, respectively, under reflux of anhydrous acetone containing potassium carbonate for $5 \mathrm{~h} .{ }^{17}$ The second step consists of the condensation reactions of these compounds with 1.2 equivalents of neat $N, N$-dimethylformamide dimethylacetal (DMFDMA) under reflux for $10 \mathrm{~h}$. After removal of the excess reagent, 3-dimethylamino-2-(3,5-dimethyl-1 $\mathrm{H}$-1pyrazolyl)-1-phenyl-2-propen-1-one 3a (in 92\% yield) and 3-dimethylamino-2-(3,5-dimethyl-1H-1-pyrazolylmethyl)1-phenyl-2-propen-1-one 3b (in 98\% yield) were obtained.

Although carbanions resulting from proton abstraction from $\mathrm{N}$-alkylpyrazoles might also be stabilized by the electron-attracting inductive effect of the ring nitrogen, the reactivity of such a species towards $\mathrm{N}, \mathrm{N}$-dimethylformamide dimethylacetal was demonstrated. The structure of the compounds was determined by ${ }^{1} \mathrm{H}$ NMR and ${ }^{13} \mathrm{C}$ NMR spectroscopy, MS espectrometry, and HRMS analysis. Moreover, X-ray crystallography data confirmed the structure of compound $\mathbf{3 b}$. By carrying out a single crystal $\mathrm{X}$-ray analysis, $E$-configutation of the products was unequivocaly determined, showing that a single isomer was obtained (Figure 1).

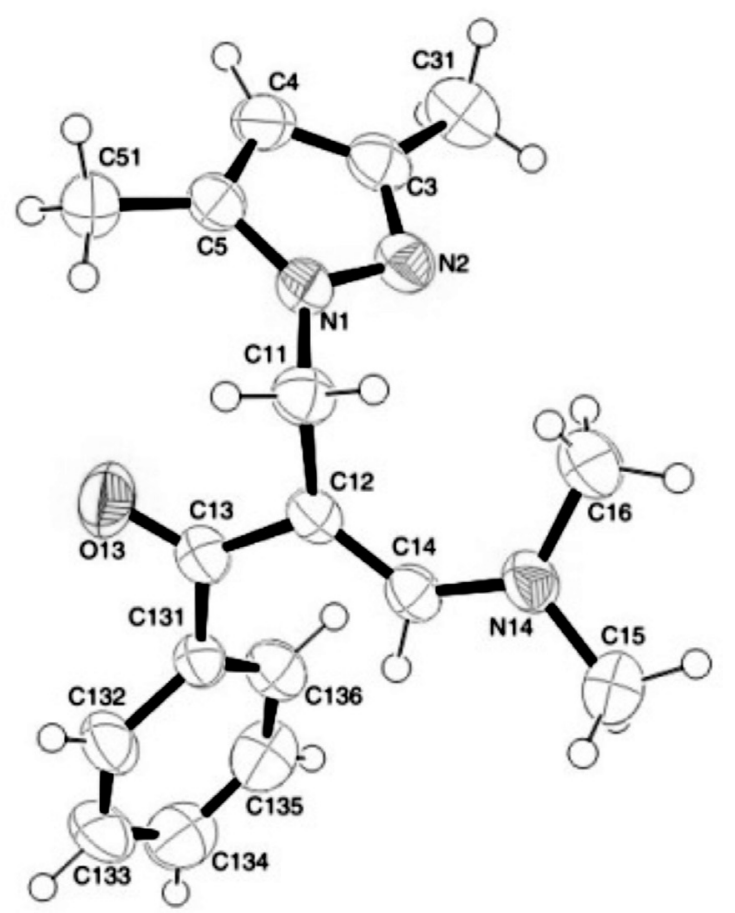

Figure 1. ORTEP of the compound $\mathbf{3 b}$.
In the last step, the $\beta$-dimethylaminoenones were converted to 1,4 '-bipyrazoles by treatment with hydrazine monohydrate, tert-butylhydrazine hydrochloride, phenylhydrazine hydrochloride and carboxymethylhydrazine. When the $\beta$-dimethylaminoenones 3a-b reacted with hydrazine monohydrate in ethanol at room temperature for $5 \mathrm{~h}$, the corresponding 1,4'-bipyrazoles $4 \mathbf{a}$ (in $71 \%$ yield) and $\mathbf{4 b}$ (in $80 \%$ yield) were obtained; and when reacted with tert-butylhydrazine hydrochloride or phenylhydrazine hydrochloride in ethanol at $60{ }^{\circ} \mathrm{C}$ for 5 h, 1,4'-bipyrazoles $\mathbf{5 a - b}$ and $\mathbf{6 a - b}$ were obtained, respectively. After an initial screen of solvents and reaction temperatures, the 1,4'-bipyrazole $\mathbf{7 a}$ was synthesized by the cyclocondensation reaction of the $\beta$-dimethylaminoenone 3a with carboxymethylhydrazine and the use of one equivalent of $\mathrm{BF}_{3} \cdot \mathrm{OEt}_{2}$ in anhydrous $\mathrm{MeOH}$ under reflux for $10 \mathrm{~h}$ in $72 \%$ yield. On the other hand, the reaction of the $\beta$-dimethylaminoenone $\mathbf{3 b}$ with carboxymethylhydrazine using these conditions did not give the expected $1,4^{\prime}$ '-bipyrazole $7 \mathbf{b}$. Our preparative results are summarized in Scheme 1 and Table 1.

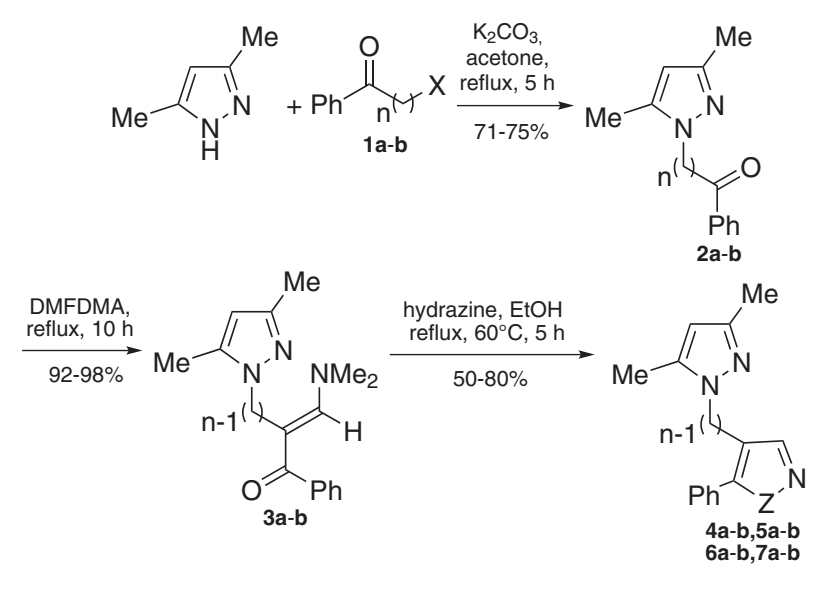

$\mathrm{Z}=\mathrm{NH}, \mathrm{N}$-tert-Bu, N-Ph, N(C(O)OMe

Scheme 1.

Table 1. Reaction conditions to synthesis of the 1,4'-bipyrazoles 4-7

\begin{tabular}{|c|c|c|c|c|}
\hline Conditions & $\mathrm{Z}$ & $\mathrm{n}$ & Product & Yield $(\%)^{\mathrm{a}}$ \\
\hline $\mathrm{NH}_{2} \mathrm{NH}_{2} \cdot \mathrm{H}_{2} \mathrm{O}$ & $\mathrm{NH}$ & 1 & $4 a$ & 71 \\
\hline $\mathrm{EtOH}, \mathrm{rt}, 5 \mathrm{~h}$ & $\mathrm{NH}$ & 2 & $4 b$ & 80 \\
\hline${ }^{t} \mathrm{BuNHNH}_{2} \cdot \mathrm{HCl}$, & $\mathrm{N}^{t} \mathrm{Bu}$ & 1 & $5 \mathbf{a}$ & 50 \\
\hline $\mathrm{EtOH}, 60^{\circ} \mathrm{C}, 5 \mathrm{~h}$ & $\mathrm{~N}^{t} \mathrm{Bu}$ & 2 & $5 \mathbf{b}$ & 66 \\
\hline $\mathrm{PhNHNH}_{2} \cdot \mathrm{HCl}$ & $\mathrm{NPh}$ & 1 & $6 a$ & 64 \\
\hline $\mathrm{EtOH}, 60^{\circ} \mathrm{C}, 5 \mathrm{~h}$ & $\mathrm{NPh}$ & 2 & $6 b$ & 67 \\
\hline $\mathrm{NH}_{2} \mathrm{NHCO}_{2} \mathrm{Me}$ & $\mathrm{NCO}_{2} \mathrm{Me}$ & 1 & $7 a$ & 72 \\
\hline $\mathrm{BF}_{3} \cdot \mathrm{OEt}_{2}, \mathrm{MeOH}$, reflux, $10 \mathrm{~h}$ & $\mathrm{NCO}_{2} \mathrm{Me}$ & 2 & $7 \mathrm{~b}$ & $-{ }_{-}^{\mathrm{b}}$ \\
\hline
\end{tabular}

${ }^{\mathrm{a}}$ Yield of isolated products; ${ }^{\mathrm{b}}$ Product was not identified. 


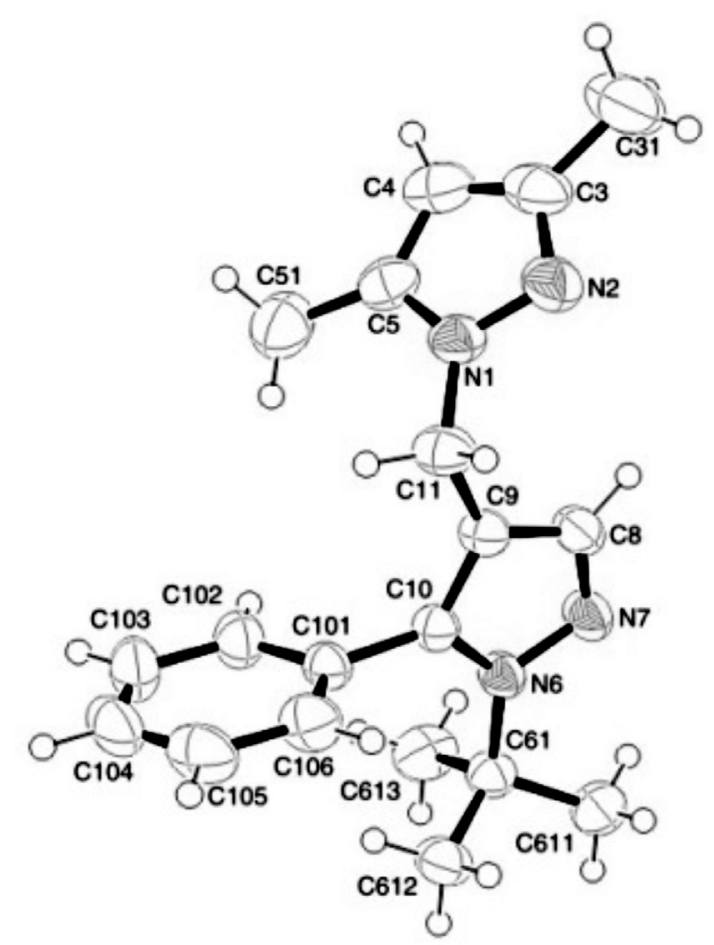

Figure 2. ORTEP of the compound $\mathbf{5 b}$.

The highly regioselective cyclocondensation reactions of the $\beta$-dimethylaminoenones $\mathbf{3 a - b}$ with different hydrazines gave 1,5-regioisomer rather than the potential 1,3-regioisomer. Moreover, the regiochemistry of the compound $\mathbf{5 b}$ was unequivocally determined by single crystal X-ray diffraction data, ${ }^{18}$ confirming the formation of the 1,5-regioisomer as a single product (Figure 2).

Thus, the $\beta$-dimethylaminoenones $\mathbf{3 a - b}$ reacted with different hydrazines to afford, in each case, only one of the two possible regioisomers. Similarly, when the $\beta$-dimethylaminoenone 3a reacted with hydroxylamine hydrochloride in ethanol at $60{ }^{\circ} \mathrm{C}$ for $5 \mathrm{~h}$, the 1,5-regioisomer of the 1,4'-bipyrazoylisoxazole (8a) was obtained with high regioselectivity in $61 \%$ yield. When the $\beta$-dimethylaminoenone $\mathbf{3 b}$ reacted with hydroxylamine hydrochloride in ethanol at $60{ }^{\circ} \mathrm{C}$ for $5 \mathrm{~h}$, the 1,5-regioisomer of the 1,4'-bipyrazoylisoxazole $(\mathbf{8 b})$ was obtained in $50 \%$ yield. Therefore, we can say that the cyclocondensation reactions of the $\beta$-dimethylaminoenones $\mathbf{3 a}$ and $\mathbf{3 b}$ with hydroxylamine hydrochloride in these conditions are highly regioselective in both cases. Our preparative results are summarized in Scheme 2.

The same synthetic route was repeated via the synthesis of (3,5-dimethyl-1H-1-pyrazolyl)acetone $\mathbf{2 c}$ (Scheme 3).

Thus, the compound $\mathbf{2 c}$ was synthesized by the alkylation reaction of 3,5-dimethyl- $1 H$-pyrazole with chloroacetone $\mathbf{1 c}$ in the presence of triethylamine under

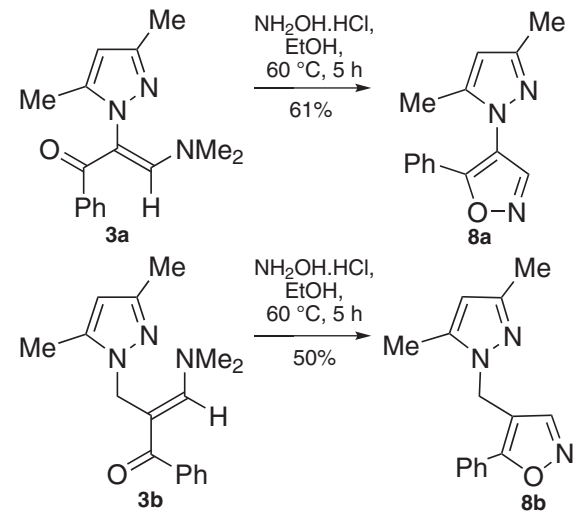

Scheme 2 .

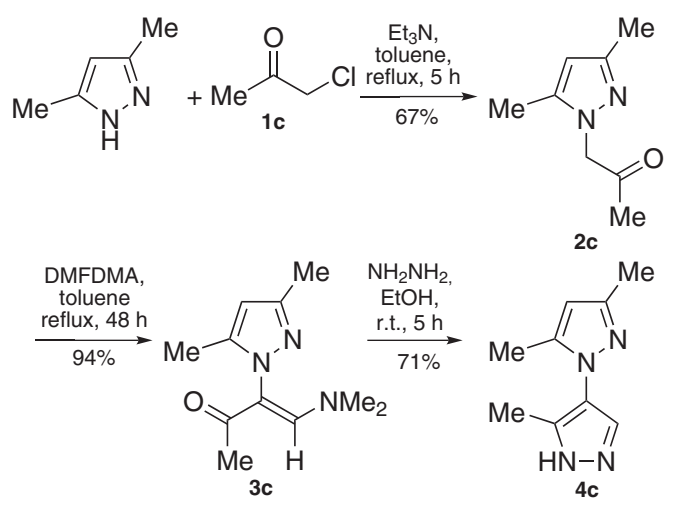

Scheme 3.

reflux of anhydrous toluene for $5 \mathrm{~h}$ in $67 \%$ yield. Then the compound $2 \mathrm{c}$ condensed with 1.2 equivalents of neat DMFDMA under reflux of toluene for $48 \mathrm{~h}$. After removal of the excess reagent, the 4-dimethylamino-3(3,5-dimethyl-1H-1-pyrazolyl)-3-buten-2-one 3c was obtained in $94 \%$ yield. Although the condensation reaction with two equivalents of DMFDMA may in theory lead to a mixture of $\mathbf{3 c}$ and $\mathbf{3} \mathbf{c}$ ' (Figure 3), in our experiment only $\mathbf{3 c}$ was formed. The ${ }^{1} \mathrm{H}$ NMR spectra of $\mathbf{3 c}$ revealed the presence of a singlet corresponding to methyl at $\delta 2.25$, and the absence of the two olefinic doublets, which would be observed in the ${ }^{1} \mathrm{H}$ NMR spectra of $\mathbf{3 c}$ '. The $\beta$-dimethylaminoenone $\mathbf{3 c}$ was used as starting material for the synthesis of the 1,4'-bipyrazole $\mathbf{4} \mathbf{c}$ through the cyclocondensation reaction with hydrazine monohydrate in ethanol at room temperature for $5 \mathrm{~h}$ resulting in the product with $71 \%$ yield.

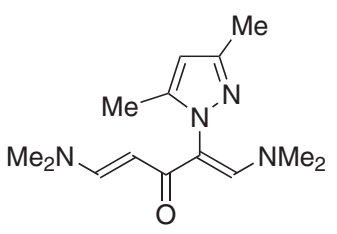

Figure 3. Theoretical product from dicondensantion. 


\section{Conclusions}

The $\beta$-dimethylaminoenones 3a-c were synthesized via highy regioselective condensation reactions. A convenient synthetic route to 1,4'-biazoles, containing a variety of neutral, electron-donating and electron-withdrawing substituents, by a simple procedure from the $\beta$-dimethylaminoenones $\mathbf{3 a - c}$ is now available. Studies on the syntheses of other heterocyclic rings from $\mathbf{3 a - c}$ are in progress.

\section{Experimental}

Unless otherwise indicated, all common reagents and solvents were used as obtained from commercial suppliers without further purification. The indicated solvents were dried and purified according to recommended procedures. ${ }^{19}$ The ${ }^{1} \mathrm{H}$ and ${ }^{13} \mathrm{C}$ NMR spectra were acquired on a Bruker DPX 200 or Bruker DPX 400 spectrometer $\left({ }^{1} \mathrm{H}\right.$ at $200.13 \mathrm{MHz}$ or $400.13 \mathrm{MHz}$ and ${ }^{13} \mathrm{C}$ at $50.32 \mathrm{MHz}$ or $100.61 \mathrm{MHz}$, respectively), in $5 \mathrm{~mm}$ tube, and $\mathrm{CDCl}_{3}$ was used as solvent, containing TMS as internal standard at $298 \mathrm{~K}$ (digital resolution of $\pm 0.01 \mathrm{ppm}$ ). The mass spectra were registered in a HP 5973 MSD connected to a HP 6890 GC and interfaced by a Pentium PC. The GC was equipped with a split-splitless injector, autosampler, cross-linked to a HP-5 capillary column ( $30 \mathrm{~m}, 0.32 \mathrm{~mm}$ of internal diameter), and helium as carrier gas. The high-resolution mass spectrum were obtained from a MicroTOF Ic Bruker Daltonics (Instituto de Química, Universidade de São Paulo, Brazil). The melting points were measure using a Microquímica MQAPF 301. The melting points were not corrected.

\section{General procedure for synthesis of $\mathbf{2} \boldsymbol{a}-\boldsymbol{b}$}

A mixture of 2-bromoacetophenone $\mathbf{1 a}$ or 3-bromopropiophenone $\mathbf{1 b}$ (20 mmol), 3,5-dimethyl- $1 \mathrm{H}$ pirazole $(20 \mathrm{mmol})$ and potassium carbonate $(20 \mathrm{mmol})$ was stirred at reflux temperature of anhydrous acetone $(20 \mathrm{~mL})$ for $5 \mathrm{~h}$. Then the mixture was filtered, extracted with dichloromethane $(20 \mathrm{~mL})$ and washed with water $(3 \times 20 \mathrm{~mL})$. The organic layer was dried with anhydrous magnesium sulfate, filtered, and the solvent was evaporated under reduced pressure. The products $\mathbf{2} \mathbf{a}-\mathbf{b}$ were purified by recrystallization in hexane as solvent.

\section{2-(3,5-Dimethyl-1H-1-pyrazolyl)acetophenone (2a)}

mp 76-79 ${ }^{\circ} \mathrm{C}$ (from hexane) (lit. ${ }^{17}$ 90-91 ${ }^{\circ} \mathrm{C}$ ); ${ }^{1} \mathrm{H}$ NMR $\left(400 \mathrm{MHz}, \mathrm{CDCl}_{3} / \mathrm{TMS}, 25^{\circ} \mathrm{C}\right) \delta 2.15(\mathrm{~s}, 3 \mathrm{H}, \mathrm{Me}), 2.23$ (s, $3 \mathrm{H}, \mathrm{Me}$ ), 5.45 (s, $2 \mathrm{H}, \mathrm{CH}_{2}$ ), 5.90 (s, 1 H, CH), 7.49 (t, $\left.J_{\mathrm{H}, \mathrm{H}}=7.7 \mathrm{~Hz}, 2 \mathrm{H}, \mathrm{Ph}\right), 7.61\left(\mathrm{t}, J_{\mathrm{H}, \mathrm{H}}=7.1 \mathrm{~Hz}, 1 \mathrm{H}, \mathrm{Ph}\right.$ ), $7.97\left(\mathrm{~d}, J_{\mathrm{H}, \mathrm{H}}=7.4 \mathrm{~Hz}, 2 \mathrm{H}, \mathrm{Ph}\right) \mathrm{ppm} ;{ }^{13} \mathrm{C}$ NMR $(100 \mathrm{MHz}$,
$\left.\mathrm{CDCl}_{3} / \mathrm{TMS}, 25^{\circ} \mathrm{C}\right) \delta 10.9(\mathrm{Me}), 13.4(\mathrm{Me}), 55.2\left(\mathrm{CH}_{2}\right)$, 105.8, 140.4, 148.2 (1H-1-pyrazolyl), 128.0, 128.8, 129.6, $133.1(\mathrm{Ph}), 192.7(\mathrm{CO}) \mathrm{ppm}$; MS (EI, $70 \mathrm{eV}) \mathrm{m} / z 214\left(\mathrm{M}^{+}\right.$, 5\%), 199 (2), 185 (100); HRMS (ESI+) $m / z[\mathrm{M}+\mathrm{H}]^{+}$calc. for $\mathrm{C}_{13} \mathrm{H}_{14} \mathrm{~N}_{2} \mathrm{O}$ : 215.1184; found: 215.1185 .

\section{3-(3,5-Dimethyl-1H-1-pyrazolyl)propiofenona (2b)}

mp 59-62 ${ }^{\circ} \mathrm{C}$ (from hexane); ${ }^{1} \mathrm{H}$ NMR $(400 \mathrm{MHz}$, $\left.\mathrm{CDCl}_{3} / \mathrm{TMS}, 25^{\circ} \mathrm{C}\right) \delta 2.19(\mathrm{~s}, 3 \mathrm{H}, \mathrm{Me}), 2.29(\mathrm{~s}, 3 \mathrm{H}, \mathrm{Me})$, $3.56\left(\mathrm{t}, J_{\mathrm{H}, \mathrm{H}}=6.8 \mathrm{~Hz}, 2 \mathrm{H}, \mathrm{CH}_{2}\right), 4.37\left(\mathrm{t}, J_{\mathrm{H}, \mathrm{H}}=6.8 \mathrm{~Hz}, 2\right.$ $\left.\mathrm{H}, \mathrm{CH}_{2}\right), 5.74(\mathrm{~s}, 1 \mathrm{H}, \mathrm{CH}), 7.44\left(\mathrm{t}, J_{\mathrm{H}, \mathrm{H}}=8.4 \mathrm{~Hz}, 2 \mathrm{H}, \mathrm{Ph}\right)$, $7.55\left(\mathrm{t}, J_{\mathrm{H}, \mathrm{H}}=7.4 \mathrm{~Hz}, 1 \mathrm{H}, \mathrm{Ph}\right), 7.93\left(\mathrm{~d}, J_{\mathrm{H}, \mathrm{H}}=7.1 \mathrm{~Hz}, 2 \mathrm{H}\right.$, $\mathrm{Ph}) \mathrm{ppm} ;{ }^{13} \mathrm{C}$ NMR $\left(50 \mathrm{MHz}, \mathrm{CDCl}_{3} / \mathrm{TMS}, 25^{\circ} \mathrm{C}\right) \delta 10.9$ (Me), $13.3(\mathrm{Me}), 38.6\left(\mathrm{CH}_{2}\right), 42.8\left(\mathrm{CH}_{2}\right)$, 104.6, 139.1, 147.5, (1H-1-pyrazolyl), 127.9, 128.4, 133.2, $136.2(\mathrm{Ph})$, 197.6 (CO) ppm; MS (EI, $70 \mathrm{eV}) \mathrm{m} / z, 228\left(\mathrm{M}^{+}, 14 \%\right), 123$ (100); HRMS (ESI+) $m / z,[\mathrm{M}+\mathrm{H}]^{+}$calc. for $\mathrm{C}_{14} \mathrm{H}_{16} \mathrm{~N}_{2} \mathrm{O}$ : 229.1341; found: 229.1343 .

\section{Procedure for synthesis of $\mathbf{2 c}$}

A mixture of chloroacetone 1c (10 mmol), 3,5-dimethyl$1 H$-pirazole $(10 \mathrm{mmol})$ and triethylamine $(10 \mathrm{mmol})$ was stirred at reflux temperature of anhydrous toluene $(3 \mathrm{~mL})$ for $5 \mathrm{~h}$. Then the mixture was extracted with dichloromethane $(10 \mathrm{~mL})$ and washed with water $(3 \times 10 \mathrm{~mL})$. The organic layer was dried with anhydrous magnesium sulfate, filtered, and the solvent was evaporated under reduced pressure. The product $\mathbf{2 c}$ was purified by recrystallization in hexane as solvent.

\section{(3,5-Dimethyl-1H-1-pyrazolyl)acetone 2c}

$\mathrm{mp} 49-51{ }^{\circ} \mathrm{C}$ (from hexane) (lit. ${ }^{20} 67-68{ }^{\circ} \mathrm{C}$ ); ${ }^{1} \mathrm{H}$ NMR $\left(200 \mathrm{MHz}, \mathrm{CDCl}_{3} / \mathrm{TMS}, 25^{\circ} \mathrm{C}\right) \delta 2.10(\mathrm{~s}, 3 \mathrm{H}, \mathrm{Me}), 2.16$ (s, $3 \mathrm{H}, \mathrm{Me}$ ), 2.22 (s, $3 \mathrm{H}, \mathrm{Me}$ ), 4.76 (s, $2 \mathrm{H}, \mathrm{CH}_{2}$ ), 5.89 (s, $1 \mathrm{H}, \mathrm{CH}) \mathrm{ppm} ;{ }^{13} \mathrm{C} \mathrm{NMR}\left(50 \mathrm{MHz}, \mathrm{CDCl}_{3} / \mathrm{TMS}, 25^{\circ} \mathrm{C}\right) \delta$ $10.9(\mathrm{Me}), 13.4(\mathrm{Me}), 26.8(\mathrm{Me}), 58.3\left(\mathrm{CH}_{2}\right), 105.9,139.8$, 148.4 (1H-1-pyrazolyl), 203.0 (CO) ppm; MS (EI, $70 \mathrm{eV}$ ) $\mathrm{m} / \mathrm{z} 152\left(\mathrm{M}^{+}, 49 \%\right), 137$ (1), 109 (100); HRMS (ESI+) $\mathrm{m} / \mathrm{z}$ $[\mathrm{M}+\mathrm{H}]^{+}$calc. for $\mathrm{C}_{8} \mathrm{H}_{12} \mathrm{~N}_{2} \mathrm{O}$ : 153.1028; found: 153.1023 .

General procedure for synthesis of $\beta$-dimethylaminoenones $3 a-b$

2-(3,5-Dimethyl-1H-1-pyrazolyl)acetophenone 2a or 3-(3,5-dimethyl-1H-1-pyrazolyl)propiophenone $\mathbf{2 b}$ $(10 \mathrm{mmol})$ was stirred at reflux temperature of DMFDMA (12 mmol) for $10 \mathrm{~h}$. Then the mixture was submitted at reduced pressure for $1 \mathrm{~h}$. The product 3a was purified by filtration in a column chromatographic silica gel with dichloromethane as an eluent, and the product $\mathbf{3 b}$ was purified by recrystallization in ethyl acetate as solvent. 
(Z)-3-Dimethylamino-2-(3,5-dimethyl-1H-1-pyrazolyl)-1phenyl-2-propen-1-one ( $3 \boldsymbol{a})$

Oil; ${ }^{1} \mathrm{H}$ NMR $\left(200 \mathrm{MHz}, \mathrm{CDCl}_{3} / \mathrm{TMS}, 25{ }^{\circ} \mathrm{C}\right) \delta 1.94$ (s, $3 \mathrm{H}, \mathrm{Me}$ ), 2.26 (s, $3 \mathrm{H}, \mathrm{Me}$ ), 2.35 (s, $3 \mathrm{H}, \mathrm{NMe}$ ), 3.11 (s, $3 \mathrm{H}, \mathrm{NMe}$ ), 5.79 (s, $1 \mathrm{H}, \mathrm{CH}), 7.22-7.35$ (m, $3 \mathrm{H}, \mathrm{Ph}$ ), 7.41-4.46 (m, $2 \mathrm{H}, \mathrm{Ph}), 7.61$ (s, $1 \mathrm{H}, \mathrm{CH}) \mathrm{ppm} ;{ }^{13} \mathrm{C} \mathrm{NMR}$ $\left(50 \mathrm{MHz}, \mathrm{CDCl}_{3} / \mathrm{TMS}, 25^{\circ} \mathrm{C}\right) \delta 11.3(\mathrm{Me}), 13.7(\mathrm{Me})$, $36.4\left(\mathrm{NMe}_{2}\right), 105.4,143.3,148.3$ (1H-1-pyrazolyl), 106.9 (C2), 109.9 (CH), 127.8, 127.9, 129.9, $139.7(\mathrm{Ph}), 190.3$ (CO) ppm; MS (EI, $70 \mathrm{eV}) \mathrm{m} / z 269\left(\mathrm{M}^{+}, 7 \%\right), 268(8)$, 239 (40), 188 (100); HRMS (ESI+) $\mathrm{m} / z[\mathrm{M}+\mathrm{H}]^{+}$calc. for $\mathrm{C}_{16} \mathrm{H}_{19} \mathrm{~N}_{3} \mathrm{O}$ : 270.1606; found: 270.1614 .

(E)-3-Dimethylamino-2-(3,5-dimethyl-1H-1pyrazolylmethyl)-1-phenyl-2-propen-1-one ( $3 \boldsymbol{b})$

mp 115-118 ${ }^{\circ} \mathrm{C}$ (from ethyl acetate); ${ }^{1} \mathrm{H}$ NMR $\left(200 \mathrm{MHz}, \mathrm{CDCl}_{3} / \mathrm{TMS}, 25^{\circ} \mathrm{C}\right) \delta 2.19(\mathrm{~s}, 3 \mathrm{H}, \mathrm{Me}), 2.39$ (s, $3 \mathrm{H}, \mathrm{Me}$ ), 3.23 (s, $6 \mathrm{H}, \mathrm{NMe}_{2}$ ), 5.12 (s, $2 \mathrm{H}, \mathrm{CH}_{2}$ ), 5.73 (s, $1 \mathrm{H}, \mathrm{CH}), 7.13(\mathrm{~s}, 1 \mathrm{H}, \mathrm{CH}), 7.32-7.37(\mathrm{~m}, 3 \mathrm{H}, \mathrm{Ph})$, 7.41-7.45 (m, $2 \mathrm{H}, \mathrm{Ph}) \mathrm{ppm} ;{ }^{13} \mathrm{C} \mathrm{NMR}\left(50 \mathrm{MHz}, \mathrm{CDCl}_{3} /\right.$ TMS, $\left.25{ }^{\circ} \mathrm{C}\right) \delta 11.2(\mathrm{Me}), 13.6(\mathrm{Me}), 41.7\left(\mathrm{CH}_{2}\right), 43.8$ $\left(\mathrm{NMe}_{2}\right), 104.4,141.3,146.6$ (1H-1-pyrazolyl), $105.3(\mathrm{C} 2)$, 127.7, 128.2, 129.3, $139.8(\mathrm{Ph}), 157.2(\mathrm{CH}), 195.8(\mathrm{CO})$ ppm; MS (EI, $70 \mathrm{eV}) \mathrm{m} / z, 283\left(\mathrm{M}^{+}, 7 \%\right), 268$ (8), 239 (40), 188 (100); HRMS (ESI+) $\mathrm{m} / z$ [M $+\mathrm{Na}]^{+}$calc. for $\mathrm{C}_{17} \mathrm{H}_{21} \mathrm{~N}_{3} \mathrm{ONa}$ : 306.1583; found: 306.1585 .

Experimental procedure for synthesis of $\beta$-dimethylaminoenone $3 \mathrm{c}$

A mixture of (3,5-dimethyl-1H-1-pyrazolyl)acetone 2c (3 mmol) and DMFDMA (3.6 mmol) was stirred at reflux temperature of anhydrous toluene $(1 \mathrm{~mL})$ for $48 \mathrm{~h}$. Then the mixture was submitted at reduced pressure for $1 \mathrm{~h}$. The product $\mathbf{3 c}$ was purified by filtration in a column chromatographic silica gel with dichloromethane as an eluent.

(Z)-4-Dimethylamino-3-(3,5-dimethyl-1H-1-pyrazolyl)-3buten-2-one $(3 \mathrm{c})$

Oil; ${ }^{1} \mathrm{H}$ NMR (200 MHz, CDCl $\left./ \mathrm{TMS}, 25{ }^{\circ} \mathrm{C}\right) \delta 1.78$ (s, $3 \mathrm{H}, \mathrm{Me}$ ), 2.10 (s, $3 \mathrm{H}, \mathrm{Me}$ ), 2.25 (s, $3 \mathrm{H}, \mathrm{Me}$ ), 2.29 (s, $3 \mathrm{H}, \mathrm{NMe}$ ), 3.11 (s, $3 \mathrm{H}, \mathrm{NMe}$ ), 5.91 (s, $1 \mathrm{H}, \mathrm{CH}), 7.65$ (s, $1 \mathrm{H}, \mathrm{CH}) \mathrm{ppm} ;{ }^{13} \mathrm{C}$ NMR $\left(100 \mathrm{MHz}, \mathrm{CDCl}_{3} / \mathrm{TMS}\right.$, $\left.25^{\circ} \mathrm{C}\right) \delta 11.4(\mathrm{Me}), 13.6(\mathrm{Me}), 24.5(\mathrm{Me}), 36.2(\mathrm{NMe})$, 47.3 (NMe), 105.2, 142.9, 148.8 (1H-1-pyrazolyl), 105.4 (C3), $109.3(\mathrm{CH}), 189.6$ (CO) ppm; MS (EI, $70 \mathrm{eV}$ ) m/z $207\left(\mathrm{M}^{+}, 53 \%\right), 192$ (100), 164 (31), 135 (16); HRMS (ESI+) $\mathrm{m} / z[\mathrm{M}+\mathrm{H}]^{+}$calc. for $\mathrm{C}_{11} \mathrm{H}_{17} \mathrm{~N}_{3} \mathrm{O}: 208.1450$; found: 208.1432 .
General Procedure for synthesis of 1,4'-bipyrazoles $\mathbf{4 a - c}$

A mixture of $\beta$-dimethylaminoenone $\mathbf{3 a}, \mathbf{3 b}$ or $\mathbf{3 c}$ $(1 \mathrm{mmol})$, hydrazine monohydrate $(1.2 \mathrm{mmol})$ and ethanol $(1 \mathrm{mmol})$ was stirred at room temperature for $5 \mathrm{~h}$. Then the mixture was extracted with dichloromethane $(3 \mathrm{~mL})$ and washed with water $(3 \times 3 \mathrm{~mL})$. The organic layer was dried with anhydrous magnesium sulfate, filtered, and the solvent was evaporated under reduced pressure. The products $\mathbf{4 a - c}$ were purified by filtration in a column chromatographic silica gel with dichloromethane as an eluent.

3,5-Dimethyl-5'-phenyl-1H-1,4'-bipyrazole (4a)

Oil; ${ }^{1} \mathrm{H}$ NMR $\left(200 \mathrm{MHz}, \mathrm{CDCl}_{3} / \mathrm{TMS}, 25^{\circ} \mathrm{C}\right) \delta 1.88$ (s, $3 \mathrm{H}, \mathrm{Me}$ ), 2.31 (s, $3 \mathrm{H}, \mathrm{Me}$ ), 5.95 (s, $1 \mathrm{H}, \mathrm{CH}$ ), 7.25-7.35 (m, $6 \mathrm{H}, \mathrm{Ph}$ and $\mathrm{NH}), 7.67$ (s, $1 \mathrm{H}, \mathrm{CH}) \mathrm{ppm} ;{ }^{13} \mathrm{C} \mathrm{NMR}$ $\left(100 \mathrm{MHz}, \mathrm{CDCl}_{3} / \mathrm{TMS}, 25{ }^{\circ} \mathrm{C}\right) \delta 11.1(\mathrm{Me}), 13.4(\mathrm{Me})$, $105.8,125.7,128.2,128.6,128.6,129.7,132.3,141.9$, 143.2, $149.2(2 \times 1 H$-1-pyrazolyl and Ph) ppm; MS (EI, $70 \mathrm{eV}) \mathrm{m} / \mathrm{z} 238\left(\mathrm{M}^{+}, 49 \%\right), 223$ (100); HRMS (ESI+) $\mathrm{m} / \mathrm{z}$ $[\mathrm{M}+\mathrm{H}]^{+}$calc. for $\mathrm{C}_{14} \mathrm{H}_{14} \mathrm{~N}_{4}: 239.1296$; found: 239.1292 .

1-(3,5-Dimethyl-1H-1-pyrazolyl)-1-(5-phenyl-1H-4pyrazolyl)methane $(\mathbf{4 b})$

Oil; ${ }^{1} \mathrm{H}$ NMR $\left(200 \mathrm{MHz}, \mathrm{CDCl}_{3} / \mathrm{TMS}, 25^{\circ} \mathrm{C}\right) \delta 1.97$ (s, $3 \mathrm{H}, \mathrm{Me}$ ), 2.19 (s, $3 \mathrm{H}, \mathrm{Me}$ ), 5.14 (s, $2 \mathrm{H}, \mathrm{CH}_{2}$ ), 5.76 (s, $1 \mathrm{H}$, $\mathrm{CH}), 7.14$ (br s, $1 \mathrm{H}, \mathrm{NH}), 7.26-7.34(\mathrm{~m}, 4 \mathrm{H}, \mathrm{Ph}$ and $\mathrm{CH}$ ), 7.41-7.46 (m, $2 \mathrm{H}, \mathrm{Ph}) \mathrm{ppm} ;{ }^{13} \mathrm{C}$ NMR $\left(100 \mathrm{MHz}, \mathrm{CDCl}_{3} /\right.$ TMS, $\left.25^{\circ} \mathrm{C}\right) \delta 10.7(\mathrm{Me}), 13.3(\mathrm{Me}), 43.5\left(\mathrm{CH}_{2}\right), 105.2$, 114.6, 127.8, 127.9, 128.1, 128.5, 131.4, 133.7, 138.8, 147.4 (2' 1H-1-pyrazolyl and Ph) ppm; MS (EI, $70 \mathrm{eV}$ ) $\mathrm{m} / \mathrm{z} 252\left(\mathrm{M}^{+}, 41 \%\right), 223(1), 157$ (100); HRMS (ESI+) $\mathrm{m} / \mathrm{z}$ $[\mathrm{M}+\mathrm{H}]^{+}$calc. for $\mathrm{C}_{15} \mathrm{H}_{16} \mathrm{~N}_{4}: 253.1453$; found: 253.1455 .

\section{3,5-Dimethyl-5'-methyl-1H-1,4'-bipyrazole (4c)}

Oil; ${ }^{1} \mathrm{H}$ NMR $\left(400 \mathrm{MHz}, \mathrm{CDCl}_{3} / \mathrm{TMS}, 25^{\circ} \mathrm{C}\right) \delta 2.12$ (s, $3 \mathrm{H}, \mathrm{Me}$ ), 2.15 (s, $3 \mathrm{H}, \mathrm{Me}$ ), 2.28 (s, $3 \mathrm{H}, \mathrm{Me}$ ), 5.95 (s, $1 \mathrm{H}$, $\mathrm{CH}), 7.55$ (s, $1 \mathrm{H}, \mathrm{CH}) \mathrm{ppm} ;{ }^{13} \mathrm{C}$ NMR $\left(100 \mathrm{MHz}, \mathrm{CDCl}_{3} /\right.$ TMS, $\left.25{ }^{\circ} \mathrm{C}\right) \delta 9.6(\mathrm{Me}), 11.3(\mathrm{Me}), 13.4(\mathrm{Me}), 105.3$, 105.3, 141.4, 141.4, 148.9, $148.9(2 \times 1 H$-1-pyrazolyl $)$ ppm; MS (EI, $70 \mathrm{eV}) \mathrm{m} / \mathrm{z}, 176$ (M+1 100\%), 161 (6), 148 (8), 134 (24), 81 (15), 66 (12); HRMS (ESI+) $\mathrm{m} / z[\mathrm{M}+\mathrm{H}]^{+}$ calc. for $\mathrm{C}_{9} \mathrm{H}_{12} \mathrm{~N}_{4}$ : 177.1140 ; found: 177.1137 .

General procedure for synthesis of 1,4'-biazoles $\mathbf{5} \boldsymbol{a}-\boldsymbol{b}$, $\mathbf{6} \boldsymbol{a}-\boldsymbol{b}$ and $\mathbf{8} \boldsymbol{a}-\boldsymbol{b}$

A mixture of $\beta$-dimethylaminoenone $\mathbf{3 a}$ or $\mathbf{3 b}(1 \mathrm{mmol})$, tert-butylhydrazine hydrochloride, phenylhydrazine hydrochloride or hydroxylamine hydrochloride $(1.2 \mathrm{mmol})$ and ethanol was stirred at $60^{\circ} \mathrm{C}$ for $5 \mathrm{~h}$. Then the mixture 
was extracted with dichloromethane $(3 \mathrm{~mL})$ and washed with water $(3 \times 3 \mathrm{~mL})$. The organic layer was dried with anhydrous magnesium sulfate, filtered, and the solvent was evaporated under reduced pressure. The oil products were purified by filtration in a column chromatographic silica gel with dichloromethane as an eluent, and the product $\mathbf{5 b}$ was purified by recrystallization in hexane as solvent.

1 '-(tert-Butyl)-3,5-dimethyl-5'-phenyl-1H-1,4'-bipyrazole $(5 a)$

Oil; ${ }^{1} \mathrm{H}$ NMR (400 MHz, $\left.\mathrm{CDCl}_{3} / \mathrm{TMS}, 25{ }^{\circ} \mathrm{C}\right) \delta$ $1.48(\mathrm{~s}, 9 \mathrm{H}, t \mathrm{Bu}), 2.14(\mathrm{~s}, 3 \mathrm{H}, \mathrm{Me}), 2.24(\mathrm{~s}, 3 \mathrm{H}$, $\mathrm{Me}), 5.52(\mathrm{~s}, 1 \mathrm{H}, \mathrm{CH}), 7.31(\mathrm{~m}, 5 \mathrm{H}, \mathrm{Ph}), 7.62(\mathrm{~s}, 1 \mathrm{H}$, $\mathrm{CH}) \mathrm{ppm} ;{ }^{13} \mathrm{C}$ NMR $\left(100 \mathrm{MHz}, \mathrm{CDCl}_{3} / \mathrm{TMS}, 25^{\circ} \mathrm{C}\right) \delta$ $10.9(\mathrm{Me}), 13.3(\mathrm{Me}), 30.9,68.7(t \mathrm{Bu}), 105.9,127.8$, $128.1,128.3,128.9,129.9,130.7,133.1,133.9,148.3$ $(2 \times 1 H$-1-pyrazolyl and Ph) ppm; MS (EI, $70 \mathrm{eV}) \mathrm{m} / \mathrm{z}$ $294\left(\mathrm{M}^{+}, 100 \%\right), 279$ (2), 237 (29), 223 (83), 209 (6), 143 (6); HRMS (ESI+) $m / z[\mathrm{M}+\mathrm{H}]^{+}$calc. for $\mathrm{C}_{18} \mathrm{H}_{22} \mathrm{~N}_{4}$ : 295.1922; found: 295.1920 .

1-[1-(tert-Butyl)-5-phenyl-1H-4-pyrazolyl]-1-(3,5dimethyl-1H-1-pyrazolyl)methane (5b)

mp 101-104 ${ }^{\circ} \mathrm{C}$ (from hexane); ${ }^{1} \mathrm{H}$ NMR $(400 \mathrm{MHz}$, $\left.\mathrm{CDCl}_{3} / \mathrm{TMS}, 25{ }^{\circ} \mathrm{C}\right) \delta 1.41(\mathrm{~s}, 9 \mathrm{H}, t \mathrm{Bu}), 1.85(\mathrm{~s}, 3 \mathrm{H}$, $\mathrm{Me}$ ), 2.17 (s, $3 \mathrm{H}, \mathrm{Me}), 4.70$ (s, $\left.2 \mathrm{H}, \mathrm{CH}_{2}\right), 5.67$ (s, $1 \mathrm{H}$, $\mathrm{CH}), 7.23-7.25$ (m, $2 \mathrm{H}, \mathrm{Ph}), 7.38$ (s, $1 \mathrm{H}, \mathrm{CH}), 7.39-7.42$ (m, $3 \mathrm{H}, \mathrm{Ph}$ ) ppm; ${ }^{13} \mathrm{C}$ NMR (100 MHz, $\mathrm{CDCl}_{3} / \mathrm{TMS}, 25$ $\left.{ }^{\circ} \mathrm{C}\right) \delta 10.7(\mathrm{Me}), 13.4(\mathrm{Me}), 30.9,61.2(t \mathrm{Bu}), 43.0\left(\mathrm{CH}_{2}\right)$, 104.8, 117.9, 128.1, 128.4, 128.7, 130.9, 132.4, 136.3, 138.5, $147.1(2 \times 1 H-1$-pyrazolyl and Ph) ppm; MS (EI, $70 \mathrm{eV}) \mathrm{m} / \mathrm{z} 308$ ( $\left.\mathrm{M}^{+}, 14 \%\right), 293$ (1), 251 (11), 213 (19), 157 (100); HRMS (ESI+) $\mathrm{m} / z[\mathrm{M}+\mathrm{H}]^{+}$calc. for $\mathrm{C}_{19} \mathrm{H}_{24} \mathrm{~N}_{4}$ : 309.2079; found: 309.2078.

\section{1',5'-Diphenyl-3,5-dimethyl-1H-1,4'-bipyrazole (6a)}

Oil; ${ }^{1} \mathrm{H}$ NMR (400 MHz, $\mathrm{CDCl}_{3} / \mathrm{TMS}, 25^{\circ} \mathrm{C}$ ) $\delta 1.79$ (s, $3 \mathrm{H}, \mathrm{Me}$ ), 2.28 (s, $3 \mathrm{H}, \mathrm{Me}), 5.84$ (s, $1 \mathrm{H}, \mathrm{CH}), 6.99-7.02$ (m, $2 \mathrm{H}, \mathrm{Ph}$ ), 7.19-7.24 (m, $3 \mathrm{H}, \mathrm{Ph}), 7.30-7.33$ (m, $5 \mathrm{H}, \mathrm{Ph}$ ), 7.88 (s, $1 \mathrm{H}, \mathrm{CH}) \mathrm{ppm} ;{ }^{13} \mathrm{C} \mathrm{NMR}\left(100 \mathrm{MHz}, \mathrm{CDCl}_{3} / \mathrm{TMS}\right.$, $\left.25^{\circ} \mathrm{C}\right) \delta 11.1(\mathrm{Me}), 13.6(\mathrm{Me}), 105.5,122.1,127.7,127.8$, 128.5, 128.7, 128.9, 129.0, 125.2, 138.2, 138.7, 141.5, 149.1, $139.8(2 \times 1 H$-1-pyrazolyl and $2 \times \mathrm{Ph}) \mathrm{ppm}$; MS (EI, $70 \mathrm{eV}) \mathrm{m} / \mathrm{z} 314\left(\mathrm{M}^{+}, 100 \%\right), 285$ (1); HRMS (ESI+) $\mathrm{m} / \mathrm{z}$ $[\mathrm{M}+\mathrm{H}]^{+}$calc. for $\mathrm{C}_{20} \mathrm{H}_{18} \mathrm{~N}_{4}: 315.1609$; found: 315.1609 .

\section{1-(3,5-Dimethyl-1H-1-pyrazolyl)-1-(1,5-diphenyl-1H-4- pyrazolyl)methane $(\boldsymbol{6} \boldsymbol{b})$}

Oil; ${ }^{1} \mathrm{H}$ NMR $\left(200 \mathrm{MHz}, \mathrm{CDCl}_{3} / \mathrm{TMS}, 25^{\circ} \mathrm{C}\right) \delta 1.95(\mathrm{~s}$, $3 \mathrm{H}, \mathrm{Me}$ ), 2.23 (s, $3 \mathrm{H}, \mathrm{Me}$ ), 5.06 (s, $2 \mathrm{H}, \mathrm{CH}_{2}$ ), 5.76 (s, 1
H, CH, 7.16-7.26 (m, 7 H, Ph), 7.35-7.38 (m, 3 H, Ph), 7.59 (s, $1 \mathrm{H}, \mathrm{CH}$ ) ppm; ${ }^{13} \mathrm{C} \mathrm{NMR}\left(50 \mathrm{MHz}, \mathrm{CDCl}_{3} / \mathrm{TMS}, 25^{\circ} \mathrm{C}\right)$ $\delta 10.7(\mathrm{Me}), 13.1(\mathrm{Me}), 43.1\left(\mathrm{CH}_{2}\right), 105.5,117.1,119.7$, 124.6, 127.1, 128.6, 128.7, 128.7, 128.7, 128.8, 129.4, 129.6, 129.9, $140.1(2 \times 1 H$-1-pyrazolyl and $2 \times \mathrm{Ph}) \mathrm{ppm}$; MS (EI, $70 \mathrm{eV}) \mathrm{m} / z 328\left(\mathrm{M}^{+}, 14 \%\right), 233$ (100), 219 (3); HRMS (ESI+) $m / z,[\mathrm{M}+\mathrm{H}]^{+}$calc. for $\mathrm{C}_{21} \mathrm{H}_{20} \mathrm{~N}_{4}: 329.1766$; found: 329.1769 .

4-(3,5-Dimethyl-1H-1-pyrazolyl)-5-phenylisoxazole (8a) Oil; ${ }^{1} \mathrm{H}$ NMR (200 MHz, $\left.\mathrm{CDCl}_{3} / \mathrm{TMS}, 25{ }^{\circ} \mathrm{C}\right) \delta 1.92$ (s, $3 \mathrm{H}, \mathrm{Me}), 2.31$ (s, $3 \mathrm{H}, \mathrm{Me}), 5.99(\mathrm{~s}, 1 \mathrm{H}, \mathrm{CH})$, 7.32-7.35 (m, $3 \mathrm{H}, \mathrm{Ph}), 7.40$ (m, $2 \mathrm{H}, \mathrm{Ph}), 8.74$ (s, $1 \mathrm{H}$, $\mathrm{CH}) \mathrm{ppm} ;{ }^{13} \mathrm{C} \mathrm{NMR}\left(50 \mathrm{MHz}, \mathrm{CDCl}_{3} / \mathrm{TMS}, 25^{\circ} \mathrm{C}\right) \delta 11.1$ (Me), 13.5 (Me), 106.8, 126.1, 126.8, 127.2, 128.9, 129.1, 130.3, 150.5, 156.4, $158.2(2 \times 1 H$-1-pyrazolyl and $\mathrm{Ph})$ ppm; MS (EI, $70 \mathrm{eV}$ ) m/z 239 ( $\left.\mathrm{M}^{+}, 100 \%\right), 210$ (46), 144 (44); HRMS (ESI+) $m / z[\mathrm{M}+\mathrm{H}]^{+}$calc. for $\mathrm{C}_{14} \mathrm{H}_{13} \mathrm{~N}_{3} \mathrm{O}$ : 240.1137; found: 240.1137 .

1-(3,5-Dimethyl-1H-1-pyrazolyl)-1 -(5-phenyl-4isoxazolyl)methane $(\mathbf{8 b})$

Oil; ${ }^{1} \mathrm{H}$ NMR $\left(400 \mathrm{MHz}, \mathrm{CDCl}_{3} / \mathrm{TMS}, 25{ }^{\circ} \mathrm{C}\right) \delta 2.05$ (s, $3 \mathrm{H}, \mathrm{Me}$ ), 2.21 (s, $3 \mathrm{H}, \mathrm{Me}$ ), 5.19 (s, $1 \mathrm{H}, \mathrm{CH}), 5.82$ (s, $2 \mathrm{H}, \mathrm{CH}_{2}$ ), 7.49-7.52 (m, $\left.3 \mathrm{H}, \mathrm{Ph}\right)$, 7.67-7.69 (m, $2 \mathrm{H}$, $\mathrm{Ph}), 7.96$ (s, $1 \mathrm{H}, \mathrm{CH}) \mathrm{ppm} ;{ }^{13} \mathrm{C} \mathrm{NMR}\left(50 \mathrm{MHz}, \mathrm{CDCl}_{3} /\right.$ TMS, $\left.25^{\circ} \mathrm{C}\right) \delta 10.8(\mathrm{Me}), 13.3(\mathrm{Me}), 42.2\left(\mathrm{CH}_{2}\right), 105.9$, 111.6, 125.7, 127.1, 129.0, 130.2, 138.9, 148.1, 151.1, 164.5 (1H-1-pyrazolyl, $\mathrm{Ph}$ and isoxazolyl) ppm; MS (EI, $70 \mathrm{eV}) \mathrm{m} / z 253\left(\mathrm{M}^{+}, 100 \%\right), 238$ (8), 224 (17), 158 (37); HRMS (ESI+) $m / z[\mathrm{M}+\mathrm{H}]^{+}$calc. for $\mathrm{C}_{15} \mathrm{H}_{15} \mathrm{~N}_{3} \mathrm{O}: 254.1293$; found: 254.1296 .

Experimental procedure for synthesis of 1,4'-bipyrazole 7 a

A mixture of $\beta$-dimethylaminoenone 3a $(1 \mathrm{mmol})$, carboxymethylhydrazine $(1.2 \mathrm{mmol})$ and $\mathrm{BF}_{3} \mathrm{OEt}_{2}$ $(1.2 \mathrm{mmol})$ was stirred at reflux temperature of methanol $(1 \mathrm{~mL})$ for $10 \mathrm{~h}$. Then the mixture was extracted with dichloromethane $(3 \mathrm{~mL})$ and washed with water $(3 \times \mathrm{mL})$. The organic layer was dried with anhydrous magnesium sulfate, filtered, and the solvent was evaporated under reduced pressure. The product $\mathbf{7 a}$ was purified by filtration in a column chromatographic silica gel with dichloromethane as an eluent.

3,5-Dimethyl-5'-phenyl-1'-methylcarboxyl-1H-1,4'bipyrazole (7a)

Oil; ${ }^{1} \mathrm{H}$ NMR (400 MHz, $\mathrm{CDCl}_{3} / \mathrm{TMS}, 25^{\circ} \mathrm{C}$ ) $\delta 2.07$ (s, $3 \mathrm{H}, \mathrm{Me}$ ), 2.21 (s, $3 \mathrm{H}, \mathrm{Me}$ ), 3.85 (s, $1 \mathrm{H}, \mathrm{OMe}$ ), 5.14 (s, $1 \mathrm{H}, \mathrm{CH}), 7.33$ (s, $1 \mathrm{H}, \mathrm{CH}), 7.37-7.39$ (m, $5 \mathrm{H}, \mathrm{Ph}$ ) ppm; 
${ }^{13} \mathrm{C}$ NMR (100 MHz, $\left.\mathrm{CDCl}_{3} / \mathrm{TMS}, 25{ }^{\circ} \mathrm{C}\right) \delta 11.1(\mathrm{Me})$, 13.2 (Me), 55.3 (OMe), 105.7, 126.4, 126.9, 128.0, 128.6, 128.6, 128.8, 129.4, 133.9, 148.4, (2×1H-1-pyrazolyl and $\mathrm{Ph}$ ), 155.3 (CO) ppm; MS (EI, 70 eV) m/z 296 (M+, 25\%), 281 (100), 265 (1), 237 (3); HRMS (ESI+) $m / z[\mathrm{M}+\mathrm{Na}]^{+}$ calc. for $\mathrm{C}_{16} \mathrm{H}_{16} \mathrm{~N}_{4} \mathrm{O}_{2} \mathrm{Na}$ : 319.1171; found: 319.1172 .

\section{Acknowledgments}

The autors thank the Conselho Nacional de Desenvolvimento Científico e Tecnológico em Pesquisa (CNPq/PADCT), Coordenação de Aperfeiçoamento de Pessoal de Nível Superior (CAPES) and Fundação de Amparo à Pesquisa do Estado do Rio Grande do Sul (FAPERGS) for financial support. The fellowships from CNPq, CAPES and FAPERGS are also acknowledged.

\section{References}

1. For example, there are 2292 pyrazoles and 906 isoxazoles in the MDL Drug Report (MDDR-3D, 99.2), representing 3.0\% of entries. They are $1.6 \%$ of entries in Comprehensive Medicinal Chemistry (CMC-3D 99.1). Both databases are from MDL Information Systems.

2. Penning, T. D.; Talley, J. J.; Bertenshaw, S. R.; Carter, J. S.; Collins, P. W.; Docter, S.; Graneto, M. J.; Lee, L. F.; Malecha, J. W.; Miyashiro, J. M.; Rogers, R. S.; Rogier, D. J.; Yu, S. S.; Anderson, G. D.; Burton, E. G.; Cogburn, J. N.; Gregory, S. A.; Koboldt, C. M.; Perkins, W. E.; Seibert, K.; Veenhuizen, A. W.; Zhang, Y. Y.; Isakson, P. C.; J. Med. Chem. 1997, 40, 1347.

3. Smith, J. A. S.; Wehrle, B.; Aguilar-Parrilla, F.; Limbach, H.-H.; Foces-Foces, M. C.; Cano, F. H.; Elguero, J.; Baldy, A.; Pierrot, M.; Khurshid, M. M. T.; Larcombe-McDouall, J. B.; J. Am. Chem. Soc. 1989, 111, 7304

4. Foces-Foces, C.; Echevarria, A.; Jagerovich, N.; Alkorta, I.; Elguero, J.; Langer, U.; Klein, O.; Minguet-Bonvehi, M.; Limbach, H. -H.; J. Am. Chem. Soc. 2001,123, 7898.

5. Bertolasi, V.; Gilli, P.; Ferretti, V.; Gilli, G.; Fernandez-Castaño, C.; Acta Crystallogr. Sect. B 1999, 55, 985.

6. De Paz, J. L. G.; Elguero, J.; Foces-Foces, C.; Llamas-Saiz, A. L.; Aguilar-Parrilla, F.; Klein, O.; Limbach, H. -H.; J. Chem. Soc., Perkin Trans. 1997, 2, 101.

7. Foces-Foces, C.; Hager, O.; Jagerovic, N.; Jimeno, M.L.; Elguero, J.; Chem. Eur. J. 1997, 3,121.

8. Claramunt, R. M.; Maria, M. D. S.; Forfar, I.; Aguilar-Parrilla, F.; Minguet-Bonvehi, M.; Klein, O.; Limbach, H. -H.; FocesFoces, C.; Llamas-Saiz, A.L.; Elguero, J.; J. Chem. Soc., Perkin Trans. 1997, 2, 1867.

9. Claramunt, R. M.; Sanz, D.; López, C.; Jiménez, J. A.; Magn. Res. Chem. 1997, 35,35.
10. Elguero, J.; Cano, F. H.; Foces-Foces, C.; Llamas-Saiz, A. L.; Limbach, H.- H.; Aguilar-Parrilla, F.; Claramunt, R. M.; López, C.; J. Heterocycl. Chem. 1994, 31, 695.

11. Llamas-Saiz, A. L.; Foces-Foces, C.; Elguero, J.; J. Mol. Struct. 1994, 319, 231.

12. Sellier, N.; J. Heterocycl. Chem. 1999, 36, 1291; Shironina, T. M.; Igidov, N. M.; Koz'minykh, E. N.; Kon'shina, L. O.; Kasatkina, Yu. S.; Koz'minykh, V. O.; Russ. J. Org. Chem. 2001, 37, 1555; Vicente, V.; Fruchier, A.; Elguero, J.; ARKIVOC 2004, iii, 5; Bouabdallah, I.; Touzani, R.; Zidane, I.; Ramdani, A.; Radi, S.; ARKIVOC 2006, xiv, 46; Bakavoli, M.; Feizyzadeh, B.; Rahimizadeh, M.; Tetrahedron Lett. 2006, 47, 8965;

13. Chen, H. S.; Li, Z. M.; Wang, Z. W.; Chin. Chem. Lett. 1999, $10,643$.

14. Hoz, A. de la; Díaz, A.; Elguero, J.; Jiménez, A.; Moreno, A.; Ruiz, A.; Sánches-Migallón, A.; Tetrahedron 2007, 63, 748.

15. Martins M. A. P.; Frizzo, C. P.; Moreira, D. N.; Zanatta, N.; Bonacorso H. G.; Chem. Rev. 2008, 108, 2015 and references therein; Martins, M. A. P.; Cunico, W.; Pereira, C. M. P; Flores, A. F. C.; Bonacorso H. G.; Zanatta, N.; Curr. Org. Synth. 2004, 1, 391 and references therein; Bonacorso, H. G.; Lopes, I. S.; Wastowski, A. D.; Zanatta, N.; Martins, M. A. P.; J. Fluorine Chem. 2003, 120, 29; Flores, A. F. C.; Brondani, S.; Zanatta, N.; Rosa, A.; Martins, M. A. P.; Tetrahedron Lett. 2002, 43, 8701; Flores, A. F. C.; Zanatta, N.; Rosa, A.; Brondani, S.; Martins, M. A. P.; Tetrahedron Lett. 2002, 43, 5005.

16. Martins, M. A. P.; Cunico, W.; Scapin, E.; Emmerich, D. J.; Fiss, G. F.; Rosa, F. A.; Bonacorso, H. G.; Zanatta, N.; Flores, A. F .C.; Lett. Org. Chem. 2006, 3, 358; Zanatta, N.; Flores, D. C.; Amaral, S. S.; Bonacorso, H. G.; Martins, M. A. P.; Flores, A. F. C.; Synlett 2005, 3079; Martins, M. A. P.; Pereira, C. M. P.; Cunico, W.; Moura, S.; Rosa, F. A.; Peres, R. L.; Machado, P.; Zanatta, N.; Bonacorso, H. G.; Ultrason. Sonochem. 2006, 13, 364; Martins, M. A. P.; Cunico, W.; Brondani, S. Peres, R. L.; Zimmermann, N.; Rosa, F. A.; Fiss, G. F.; Zanatta, N.; Bonacorso, H. G.; Synthesis 2006, 9, 1485; Rosa, F. A.; Machado, P.; Bonacorso, H. G.; Zanatta, N.; Martins, M. A. P.; J. Heterocyclic. Chem. 2008, 45, 879; F. A. Rosa, Machado, P.; Rossatto, M.; Vargas, P. S.; Fernandes, T. S.; Bonacorso, H. G.; Zanatta, N.; Martins, M. A. P.; Synlett 2008, 1673; Rosa, F. A.; Machado, P.; Fiss, G. F.; Vargas, P. S.; Bonacorso, H. G.; Zanatta, N.; Martins, M. A. P.; Synthesis 2008, 3639.

17. Solomons, T. W. J.; Fowler, F. W.; Calderazzo, J.; J. Am. Chem. Soc. 1965, 87, 528.

18. Crystallographic data for the structural analysis of the compounds $\mathbf{3} \mathbf{b}$ and $\mathbf{5 b}$, reported in this paper, have been deposited with the Cambridge Crystallographic Data Center (CCDC) under the numbers 680984 and 680985, respectively. Copies of the information may be obtained, free of charge, from 
The Director, CCDC, 12 Union Road, Cambridge CB2 1EZ, UK (Fax: + 441223 336033; e-mail: deposit@ccdc.cam.ac.uk, or www: http://ccdc.cam.ac.uk).

19. Perrin, D. D.; Armarego, W. L. F.; Purification of Laboratory Chemicals, Pergamon: Oxford, 1998.
20. Mohamed, M. H.; Abdel-Khalik, M. M.; Elnagdi, M. H.; J. Heterocycl. Chem. 2001, 38, 685.

Received: March 27, 2009 Web Release Date: November 12, 2009 